\title{
Modelizando el uso de las bibliotecas públicas a través de sus características: estudio comparativo entre España y Finlandia para los préstamos y las visitas
}

\author{
Pedro Lázaro-Rodríguez*, Pertti Vakkari** \\ *Universidad de Granada. Facultad de Comunicación y Documentación \\ Correo-e: pedrolr@ugr.es | ORCID iD: https://orcid.org/0000-0002-8756-0507 \\ **Universidad de Tampere, Finlandia. Facultad de Ciencias de la Comunicación \\ Correo-e: pertti.vakkari@uta.fi | ORCID iD: https://orcid.org/0000-0002-4441-5393
}

Recibido: 05-12-2017; 2a versión: 08-02-2018; Aceptado: 12-02-2018.

Cómo citar este artículo/Citation: Lázaro-Rodríguez, P.; Vakkari, P. (2018). Modelizando el uso de las bibliotecas públicas a través de sus características: estudio comparativo entre España y Finlandia para los préstamos y las visitas. Revista Española de Documentación Científica, 41 (4): e216. https://doi.org/10.3989/redc.2018.4.1544

\begin{abstract}
Resumen: El objetivo de este artículo es la modelización del uso de las bibliotecas públicas comparando los casos de España y Finlandia. Se analiza la relación a nivel de municipios entre una serie de factores tomados como características propias de las bibliotecas (presupuesto, personal, colección, etc.) y el uso de las mismas (préstamos y visitas). En un análisis por regiones, se consideran también variables del contexto socioeconómico (renta, educación, desempleo). El método para la obtención de los modelos es el análisis de regresión lineal múltiple con la técnica paso a paso. En España, los modelos a nivel de municipios explicaron el $16,8 \%$ de los préstamos y el $17,7 \%$ de las visitas, mientras que en Finlandia explicaron el $23 \%$ y el $18 \%$ respectivamente. Por tanto, para ambos países más del $75 \%$ de la variación de los préstamos y las visitas depende de otros factores no contemplados en los modelos. A nivel de regiones, ninguna de las variables del entorno socioeconómico consideradas como añadidas respecto al análisis por municipios resultó significativa. La limitación principal del estudio es la falta de datos a nivel de municipios para las variables del entorno socioeconómico consideradas en el análisis por regiones. En cuanto al valor y su originalidad, este estudio puede ser considerado como uno de los primeros sobre modelización del uso de las bibliotecas públicas españolas y también como uno de los primeros en poner en relación los resultados con otro país, en este caso Finlandia.
\end{abstract}

Palabras clave: Bibliotecas públicas; uso de bibliotecas; modelos; análisis de regresión lineal múltiple; paso a paso; préstamos; visitas; España; Finlandia.

\section{Modelling public library use by library characteristic: a comparative study between Spain and Finland for loans and visits}

\begin{abstract}
The purpose of this paper is to model the use of public libraries carrying out a comparative study between Spain and Finland. For the analysis by municipalities, it has been studied the relation between library characteristics (expenditure, staff, collection, etc.) and the use of libraries (loans and library visits). For the analysis by regions, some other socioeconomic factors such as the level of education, incomes and unemployment rate, have been considered. The method used for the modelling is the multiple lineal regression analysis using the stepwise technique. For the analysis by municipalities in Spain, models accounted for $16.8 \%$ percent of the variation in loans and $17.7 \%$ percent in library visits. For Finland, models accounted for $23 \%$ percent and $18 \%$ respectively. Thus, in both countries more than $75 \%$ percent of the variation for loans and library visits was due to factors external to the models. For the analysis by regions, none of the socioeconomic factors were significant. The lack for data about socioeconomical factors by municipalities is the main limitation. This paper can be considered one of the first studies to model the factors on the use of Spanish public libraries and the first one comparing the results with another country (Finland).
\end{abstract}

Keywords: Public libraries; library use; modelling; multiple lineal regression analysis; stepwise; loans; visits; Spain; Finland.

Copyright: (c) 2018 CSIC. Este es un artículo de acceso abierto distribuido bajo los términos de la licencia de uso y distribución Creative Commons Reconocimiento 4.0 Internacional (CC BY 4.0). 


\section{INTRODUCCIÓN}

Las bibliotecas públicas son consideradas como un servicio esencial para la comunidad. De ellas se espera y se trabaja por obtener unos beneficios. Así, y de acuerdo a estudios recientes, son uno de los servicios públicos mejor valorados por la propia comunidad (Van-Ryzin y Charbonneau, 2010), considerándose incluso como tercer lugar junto al hogar y al trabajo (Houghton y otros, 2013; Lin y otros, 2015) y siendo especialmente defendidas desde el rol social que desempeñan (Aabo y otros, 2010; Aabo y Audunson, 2012; Herrera-Viedma y López-Gijón, 2013).

El valor de las bibliotecas públicas puede entenderse así desde la relación entre el uso y los beneficios que generan en la comunidad. En ese sentido, se han llevado a cabo estudios analizando y confirmando la relación entre el uso de las bibliotecas públicas y un mayor capital social (Johnson y Griffis, 2014) y analizando la aportación positiva de las bibliotecas públicas a cuestiones del día a día, como por ejemplo la salud, la cultura, la actividad creativa, la educación, o el trabajo (Vakkari, 2014; Sei-Ching y Vakkari, 2015). En un sentido similar, existen investigaciones comparando los beneficios asociados al uso de las bibliotecas públicas en diferentes países y analizando los factores determinantes para ello (Vakkari y otros, 2014; Vakkari y otros, 2016). Al respecto, cabe resaltar la utilidad de la comparación de países en estudios sobre bibliotecas públicas, pues dicha comparación entendida desde el poner en relación lo propio con otros casos facilita entender precisamente la naturaleza de los propios servicios y las posibilidades y potencialidades para su mejora (Gould y Gomez, 2010; Ignatow, 2011; Ignatow y otros, 2012).

El objetivo general de este estudio es la modelización del uso de las bibliotecas públicas considerando los casos de España y Finlandia. Para ello, se analiza la relación de una serie de factores con el mayor uso de los servicios por medio de análisis de regresiones lineales múltiples con la técnica paso a paso. A su vez, se trata de las diferencias entre los factores considerados para los modelos entendiéndolos como características esenciales de las bibliotecas públicas. Estos factores se refieren, por ejemplo, al presupuesto, la colección, el personal, etc., y a algunas características del entorno socioeconómico. Por otro lado, el uso se entiende como los préstamos y las visitas a las bibliotecas.

En esencia, se pretende alcanzar información sobre el estado de las bibliotecas públicas españolas en torno al uso y en su relación con unos mayores beneficios para la comunidad, llevando a cabo un ejercicio de comparación a la luz de las de Finlandia.
La motivación que explica la comparación de España con Finlandia es la de que el segundo país puede ser considerado como el modelo de buenas prácticas en lo que a bibliotecas públicas y su uso entendido desde los préstamos y las visitas se refiere, tanto en el contexto europeo como en el internacional (Vakkari y otros, 2014; Vakkari y otros, 2016).

Las preguntas de investigación concretas son las siguientes:

- ¿QQué diferencias existen entre las bibliotecas públicas de España y Finlandia en lo que a características y uso se refiere?

- ¿Qué factores explican un mayor uso en ambos países?

En las siguientes dos secciones se lleva a cabo una revisión de la literatura sobre el tema tanto a nivel nacional como internacional y se presenta el diseño de la investigación concretando el nivel de análisis, las fuentes, el procesamiento de los datos, la descripción estadística de los resultados, y el método estadístico y técnica empleados. Las secciones 4 y 5 están dedicadas respectivamente a los resultados del análisis de los factores para un mayor uso de las bibliotecas públicas en España y Finlandia, y a su discusión. Por último, se dedica una sección para las conclusiones.

\section{REVISIÓN BIBLIOGRÁFICA}

La situación de las bibliotecas públicas en España puede resultar ambigua según a qué fuentes o estudios se atienda (Lázaro-Rodríguez y otros, 2018b). Tal y como afirman los autores y conviene mencionar aquí, mientras que existen estudios que las sitúan como uno de los servicios públicos más valorados (Carrión-Gútiez, 2013), los resultados del Barómetro de junio de 2016 del Centro de Investigaciones Sociológicas (CIS) apuntan en otra dirección, pues se constata que el $74,7 \%$ de la población no ha acudido a una biblioteca en el último año (CIS, 2016), siendo el resultado peor que los del Barómetro de diciembre de 2014, donde el resultado era el $70,2 \%$ (CIS, 2014). En un mismo sentido, los resultados anteriores son similares a los de la encuesta de hábitos y prácticas culturales en España del año 2014-2015 publicada por el Ministerio de Educación, Cultura y Deporte (MECD), según los cuales solo el $25,6 \%$ de la población asistió o accedió por Internet a la biblioteca en el último año. En dicha fuente, se comprueba además que la valoración media del grado de interés por la asistencia a bibliotecas resultó de 2,7 en una escala entre 0 y 10 (MECD, 2015).

Focalizando la atención en las preguntas de investigación de este estudio, son varios los trabajos sobre la investigación en biblioteconomía y documentación 
en España que prueban la escasez en la disciplina tanto del empleo de métodos estadísticos como de investigaciones sobre el tema de las bibliotecas públicas (Delgado-López-Cozar, 2002; Ferran-Ferrer y otros, 2017; Guallar y otros, 2017). Por ejemplo, en Guallar y otros (2017) se comprueba, a partir de un análisis de las principales revistas científicas españolas en el campo, que el tema de investigación que incluye a las bibliotecas públicas obtiene un 3\% respecto del total de temas en artículos de investigación. A su vez, el empleo de métodos estadísticos, que caerían en la categoría de investigación experimental, se da en el $1 \%$ del total. De ahí que se pueda concluir la escasez tanto de los métodos estadísticos en la investigación en biblioteconomía y documentación como de las investigaciones sobre bibliotecas públicas en España. Aún con todo, cabe señalar que en el año 2018, periodo no incluido en la ventana temporal de los anteriores estudios por ser posterior a la fecha de publicación de los mismos, se encuentran estudios sobre bibliotecas públicas en España en base a métodos estadísticos. Por ejemplo, cabe destacar un trabajo que plantea la relación entre las entradas (presupuesto) y las salidas entendidas como los préstamos, todo en base al cálculo del coeficiente de correlación lineal (Lázaro-Rodríguez y otros, 2018a), y otro que analiza las características, preferencias y percepciones de las personas no usuarias de las bibliotecas con técnicas univariadas y bivariadas (Fernández-Ardévol y otros, 2018).

La situación es diferente si se atiende a la investigación en el ámbito internacional. La relación entre variables geográficas, económicas, demográficas y socioeconómicas del tipo edad, género, estado civil, nivel de educación, ocupación, o nivel de ingresos, y variables referidas al uso de bibliotecas públicas, aparece como tema central en diversos trabajos y a lo largo del tiempo (Sanderson, 1950; Obokoh y Arokoyu, 1991; Yilmaz, 1998; Japzon y Gong, 2005; Koontz y otros, 2005; Uddin y otros, 2006; Burke, 2007; Keshvari y otros, 2015; Chen y otros, 2016).

En el ámbito internacional también es prolija la investigación en torno a la relación entre factores y características propias de las bibliotecas del tipo gastos, colección, habitantes por punto de servicio, etc., con el uso de las mismas entendido desde los préstamos y las visitas (Creaser y Sumsion, 1995; Kishida, 1998; Smith, 1999; Sumsion y otros, 2002; Sei-Ching y Kyung-Sun, 2008; Huysmans y Hillebrink, 2008; Kim y Yu, 2011; Lara y otros, 2015; Whitacre y Rhinesmith, 2015). Al respecto, se constata un creciente interés por las nuevas tecnologías en el análisis de la relación entre el uso de Internet y el uso de las bibliotecas públicas (Jorgensen y otros, 2001; D'Elia y otros, 2002; Uddin y otros, 2006; Vakkari, 2012).
Igualmente, en el contexto internacional destacan investigaciones en torno al uso de las bibliotecas y los beneficios asociados a dicho uso y empleando métodos y técnicas estadísticas. Así, se puede comprobar que se han desarrollado estudios basados en análisis de regresiones lineales en aras a la modelización del uso y en torno a los beneficios asociados (McClure y Bertot, 1998; Japzon y Gong, 2005; Vakkari, 2014; Vakkari y otros, 2014; Johnson y Griffis, 2014; Sei-Ching y Vakkari, 2015; Vakkari y otros, 2016). Más aún, cabe resaltar la aplicación de la técnica paso a paso en la modelización del uso de las bibliotecas públicas (Lai y Wang, 2012; Vakkari, 2014), ejemplificando y legitimando su empleo y uso en nuevas investigaciones como la que se acomete en este trabajo.

\section{DISEÑO DE LA INVESTIGACIÓN}

\subsection{Nivel de análisis y datos}

En este estudio se consideran las bibliotecas públicas de España y Finlandia a nivel de municipios y regiones. Los datos para las bibliotecas de Finlandia se han tomado de la base de datos estadística de las bibliotecas públicas finlandesas, responsabilidad de la División para la Cultura del Ministerio de Educación y Cultura de Finlandia (SYKT, 2018). En cuanto a las bibliotecas de España, los datos han sido extraídos de la página web Bibliotecas públicas españolas en cifras, responsabilidad de la Subdirección General de Coordinación Bibliotecaria del Ministerio de Educación, Cultura y Deporte de España (MECD, 2018a).

Es importante subrayar que la toma de datos en la fuente para España resultó de una dificultad considerable en comparación a la de Finlandia. Mientras que en el segundo caso los datos para todas las municipalidades pueden recuperarse desde una única búsqueda, en el caso de España se ha de acceder primero a los resultados por comunidades autónomas y desde ahí a los de cada provincia para poder extraer los de cada municipio, requiriéndose tantas acciones de exportación y unificación en un documento como provincias existen. En ese sentido, sería recomendable que la fuente de datos sobre bibliotecas públicas españolas modificase su disposición en aras a una mayor facilidad y accesibilidad a los mismos, facilitando su procesamiento en futuras investigaciones sobre el tema.

Cabe señalar que para la toma y unificación de los datos se ha utilizado el software Libreoffice Calc 5.4.1.2. Por otro lado, para todo lo relacionado a los análisis estadísticos, se ha utilizado el software IBM SPSS 24.0 Desktop Linux Client Multilingual. 


\subsection{Descripción estadística de las variables}

Las variables consideradas se enumeran a continuación. Se ha añadido entre paréntesis la abreviación con la que se hará referencia a cada variable en lo que sigue del trabajo. Dado que se pretenden comparar los resultados de las variables entre España y Finlandia, se especifica si las variables se refieren a lo mismo en sendos países y se añaden las especificaciones concretas de las posibles diferencias y matices a tener en cuenta en la comparación. Las posibles diferencias son algo a tener en cuenta respecto de la primera pregunta de investigación en torno a la comparación de los resultados de las variables en los dos países, pero no son un factor determinante en lo relacionado a la segunda pregunta de investigación sobre los factores que llevan a un mayor uso de las bibliotecas públicas en cada país. Las variables son las siguientes:

- Gastos por habitante (Gastos): se consideran los gastos para el personal y los gastos en adquisiciones. Aunque en ambos países se refiere a lo mismo, la comparación exigiría ser conscientes de la diferencia que marca la paridad del poder adquisitivo, pues puede no suponer lo mismo una unidad monetaria en un país y en otro. Además, en la fuente de datos para Finlandia se especifica que los gastos no incluyen el IVA, mientras que en la de España no aparecen especificaciones al respecto. Se puede hacer una comparación global siendo conscientes de las consideraciones anteriores.

- Colección por habitante (Colección): similar en ambos países, considerándose la colección electrónica además de los materiales convencionales. Se puede comparar.

- Adquisiciones por $\mathbf{1 0 0}$ habitantes (Adquisiciones): similar en ambos países, incluyéndose la adquisición de materiales considerados en la Colección. Se puede comparar.

- Actividades culturales por 1000 habitantes (Actividades): similar en ambos países, aunque en Finlandia se diferencia en el total entre exhibiciones y eventos culturales. Se puede comparar.

- Superficie utilizada por 1000 habitantes (Superficie): similar en ambos países, consistiendo en la superficie en metros cuadrados por 1000 habitantes. Se puede comparar.

- Personal total por $\mathbf{5 0 0 0}$ habitantes (Personal): en España se considera como total la suma de bibliotecarios profesionales, auxiliares de biblioteca, personal especializado y otro personal; en Finlandia aparece el resultado total sin especificaciones de suma. Para ambos países se considera el personal en equivalente a tiempo completo. Se puede comparar.

- Tasa de profesionalización (Profesionales): en España consiste en el porcentaje de bibliotecarios profesionales respecto al total de personal, mientras que para Finlandia se calcula poniendo en relación el personal con estudios universitarios respecto del total de personal. Para la comparación hay que tener en cuenta lo anterior.

- Habitantes por biblioteca (Habitantes): similar en ambos países, consistiendo en la población entre los puntos de servicio. Se puede comparar.

- Préstamos por habitante (Préstamos): similar en ambos países, consistiendo en el número de préstamos de los materiales considerados como Colección entre el total de habitantes. Se puede comparar.

- Visitas por habitante (Visitas): similar en ambos países, considerándose como el total de visitas entre el total de la población. Se puede comparar.

En referencia al procesamiento de los datos, se ha entendido que pueden concebirse bibliotecas sin lo referido a Adquisiciones, Actividades y Profesionales, pero no sin lo referido a Gastos, Colección, Superficie, Personal, Habitantes, y sin suma mayor de 0 considerando los Préstamos y Visitas. Así, se han eliminado los municipios con resultado 0 en las variables de Gastos, Colección, Superficie, Personal, Habitantes, y cuando la suma de Préstamos y Visitas resultaba igualmente 0 ; pero se han mantenido los municipios en que algunas de las variables referidas a Adquisiciones, Actividades y Profesionales resultaba de 0 , respetando a su vez la cláusula anterior (por ejemplo, si un municipio presentaba solo un resultado de 0 en Adquisiciones, se ha mantenido; pero si presentaba 0 en Adquisiciones y 0 en Gastos, se ha eliminado).

Con todo, la ausencia de datos ha supuesto no considerar a 368 municipios españoles del total de los 3312 con bibliotecas, resultando un tamaño para la muestra de $2944(n=2944)$. Para el caso de Finlandia, tan solo no se consideró a un municipio por falta de datos para la Superficie, resultando una muestra de $282(n=282)$. Por otro lado, para el estudio por regiones, el tamaño de la muestra en España es de $17(n=17)$ y en Finlandia de $18(n=18)$.

Los resultados o descripción estadística de las variables en cada país a nivel de municipios (Tabla I) se comentan en las siguientes líneas. 
Tabla I. Descripción estadística de las variables a nivel de municipios

\begin{tabular}{|l|c|c|c|c|c|c|}
\hline \multirow{2}{*}{ Variable } & \multicolumn{3}{|c|}{ Finlandia $\boldsymbol{n = 2 8 2}$} & \multicolumn{3}{c|}{ España $\boldsymbol{n = 2 9 4 4}$} \\
\cline { 2 - 7 } & Media & sd & \% variación sd & Media & sd & \% variación sd \\
\hline Gastos & 41,8 & 10,5 & $25,1 \%$ & 10,7 & 17,5 & $163,9 \%$ \\
\hline Colección & 11,1 & 4,8 & $43,0 \%$ & 5,0 & 5,6 & $110,4 \%$ \\
\hline Adquisiciones & 45,9 & 15,3 & $33,3 \%$ & 14,2 & 27,9 & $196,4 \%$ \\
\hline Actividades & 9,3 & 7,5 & $80,9 \%$ & 15,3 & 41,3 & $269,9 \%$ \\
\hline Superficie & 127,4 & 49,9 & $39,2 \%$ & 118,4 & 762,5 & $644,2 \%$ \\
\hline Personal & 3,9 & 1,1 & $28,4 \%$ & 2,3 & 2,5 & $110,3 \%$ \\
\hline Profesionales & 0,4 & 0,2 & $51,7 \%$ & 0,2 & 0,3 & $164,7 \%$ \\
\hline Habitantes & 6082,1 & 5340,4 & $87,8 \%$ & 6890,6 & 9941,0 & $144,3 \%$ \\
\hline Préstamos & 15,2 & 3,4 & $22,6 \%$ & 1,4 & 2,1 & $158,5 \%$ \\
\hline Visitas & 8,6 & 2,7 & $31,7 \%$ & 2,9 & 3,3 & $114,3 \%$ \\
\hline
\end{tabular}

Nota: $s d$ equivale a desviación típica; \% variación sd se obtiene de la división de sd entre la media, multiplicado por 100.

Como se puede comprobar, la media para cada variable es siempre mayor en Finlandia que en España con excepción tan solo de las variables Actividades y Habitantes (en este último caso la interpretación es también favorable a Finlandia pues en su caso habría menos habitantes por punto de servicio). En síntesis, los Gastos son 3,9 veces mayores en Finlandia que en España, la Colección 2,2 veces, las Adquisiciones 3,2, la Superficie 1,1, el Personal 1,71, los Préstamos 11,3, y las Visitas 2,96. A su vez, los Habitantes son 0,88 veces menos en Finlandia que en España. Por otro lado, las Actividades son 1,65 mayores en España que en Finlandia. Para el caso de las variables Gastos y Profesionales hay que tener en cuenta las consideraciones matizadas en la descripción estadística de las variables, y es que para una comparación completa y correcta habría que considerar respectivamente la paridad del poder adquisitivo y las diferencias en torno a los titulados superiores en Finlandia y el personal con estudios en biblioteconomía y documentación en España.

En cuanto a las desviaciones típicas, atendiendo a la columna para el porcentaje de su variación se ve claramente que los resultados para todas las variables son más homogéneos en Finlandia que en España, país en el que en todas las variables se supera el $100 \%$ de variación, llegando incluso al $644 \%$ en el caso de la variable Superficie. Estos resultados pueden interpretarse en base a la idea de que en España hay mayores diferencias y desigualdades entre las bibliotecas públicas que en Finlandia, donde los resultados para las variables analizadas son más cercanos siempre al valor medio y, por ello, más homogéneos.
Esa mayor homogeneidad entre los municipios en Finlandia se puede entender como consecuencia de la existencia de leyes nacionales en torno a las bibliotecas públicas. Concretamente, cabe destacar la Library Act de 1961, cuyos objetivos principales fueron los de superar las diferencias entre entornos rurales y urbanos y el de aumentar el uso de las bibliotecas en ambos entornos (Mäkinen, 2001). Tal y como explica el autor, ambos objetivos se consiguieron, pasándose de unos préstamos por habitante de 1,5 en 1960 a los 19,9 en 1990. Además, el crecimiento en las zonas rurales fue mayor que en las urbanas. Con todo, aunque se reconoce también que el éxito en lo relacionado a las bibliotecas se vio soportado y potenciado por el desarrollo del estado de bienestar, en general y otros factores en particular, como el sistema educativo, el aumento de la educación superior, o la liberalización general del ambiente cultural en la misma época, una ley a nivel nacional en España sobre bibliotecas similar a la Library Act de Finlandia y todo lo que supuso para este país, marca uno de los posibles caminos para el futuro en el sentido de la mejora y homogeneización para la superación de las grandes diferencias en lo referido a bibliotecas en nuestro país.

\subsection{Método estadístico de análisis: regresión lineal múltiple paso a paso}

El análisis de regresión múltiple es una técnica usada para analizar la relación entre una serie de variables independientes y una dependiente. El objetivo es utilizar las independientes conocidos sus valores para predecir el de la considerada como dependiente (Vakkari, 2014). Tal y como afirma el autor, cada variable independiente es ponderada en el 
análisis considerando el conjunto de variables independientes para asegurar la máxima predicción sobre la dependiente. Esa ponderación se refiere a la contribución relativa de cada variable independiente a la predicción de la dependiente, lo que permite conocer la contribución relativa de cada una de las primeras a la segunda y lo que facilita la interpretación de la influencia de cada variable para la predicción. Con todo, el conjunto de variables independientes ponderadas conforman el modelo de regresión, lo que se traduce en conocer la combinación lineal de variables independientes que mejor predicen a la variable dependiente (Hair y otros, 2010).

En este estudio se consideran como variables dependientes las de Préstamos por habitante y Visitas por habitante. Por ello, se calculan en total 4 modelos de regresión lineal múltiple, considerando para cada uno de los dos países cada caso de las dos variables dependientes. Así, el primer modelo es para Finlandia y la variable dependiente de los Préstamos; el segundo, para la misma variable en el caso de España; el tercero, para Finlandia y la variable de Visitas; y el cuarto para España y la variable de Visitas.

Las variables han sido consideradas para la modelización mediante la técnica paso a paso, que permite examinar la contribución de cada variable independiente al modelo de regresión. Se considera cada variable para su inclusión antes de desarrollar la ecuación, agregando primero la variable independiente con mayor contribución (Vakkari, 2014). Desde ahí, las variables independientes restantes se van seleccionando para su inclusión en función de su contribución creciente o incremental sobre las variables que ya están en la ecuación (Hair y otros, 2010).

Para el análisis se ha verificado la relación lineal de las variables y la igualdad de las varianzas mediante gráficos residuales (Vakkari, 2014), convirtiendo a logarítmicas las variables en que no se daba dicha relación lineal. Concretamente, en el análisis para Finlandia tomando como variable dependiente los Préstamos, se han convertido a logarítmicas las variables de Gastos, Adquisiciones, Actividades, Profesionales y la propia variable dependiente de Préstamos; para el mismo país pero considerando como dependiente la variable de Visitas, se han convertido a logarítmicas las variables Profesionales y Visitas. Para España, se ha verificado que tanto en el modelo considerando como variable dependiente los Préstamos como para el modelo de las Visitas, no se da relación lineal entre las variables Profesionales y Préstamos, y Profesionales y Visitas. Desde ahí, se han convertido a logarítmicas las variables de Visitas y Préstamos, y se ha mantenido como no logarítmica la de Profesionales pese a no mostrar una relación lineal con las variables dependientes debido a que en muchos casos de la muestra el resultado para la tasa de profesionalización resultó de 0 .

Respecto a la multicolinealidad de los modelos o grado en que cada variable independiente se explica mediante el conjunto de otras variables independientes (Vakkari, 2014), los resultados para el factor de inflación de la varianza (VIF) en cada uno de los 4 modelos fueron cercanos a 1 y nunca mayores que 4 (como máximo 2,174 en España y 2,980 en Finlandia). Por ello, se puede asegurar que no existe multicolinealidad y que las premisas sobre la igualdad de la varianza, linealidad y no colinealidad, han sido respetadas (Hair y otros, 2010). A su vez, cabe destacar tras un análisis de los outliers que no se ha tenido que descartar ningún caso por no ser elevado el número de los mismos. Por último, el peso de cada variable independiente a la correspondiente variable dependiente se representa con el resultado para los coeficientes beta $(\beta)$.

\subsection{Nota para un estudio por Regiones}

Como aporte añadido, en el estudio también se presenta un análisis de las bibliotecas públicas a nivel de regiones, considerando para Finlandia las bibliotecas propiamente por regiones y para España por comunidades autónomas. El análisis por regiones se añade debido a que para ciertas variables del entorno socioeconómico y educativo que se quisieron considerar en el estudio no se disponen de datos para el nivel de análisis por municipios. Estas variables son las siguientes (se presentan siguiendo el modo para las variables por municipios, añadiendo la fuente de los datos para cada país):

- Tasa de desempleo (Desempleo): en Finlandia se trata del porcentaje de población entre 15 y 74 años sin empleo. En España el intervalo va desde los 16 a los 64 años. Los datos para Finlandia se han calculado a partir de las tablas concernientes al servicio de estadísticas para el empleo de la fuente Statistics Finland's PX-Web databases (Statfin, 2018). En España, de la sección de nombre Mercado Laboral del Instituto Nacional de Estadística (INE, 2018).

- Nivel de educación (Educación): En Finlandia es el porcentaje de personas entre 25 y 65 años con tan solo el nivel básico de educación. En España, es el porcentaje de población con educación inferior a la segunda etapa de educación secundaria entre 25 y 64 años. Los datos para Finlandia se han calculado a partir de la tabla concerniente a la estructura educativa de la población en la fuente Statistics Finland's PX-Web databases (Statfin, 2018). Para España, a partir de la base de datos sobre estadísticas de educación del MECD (MECD, 2018b). 
- $\quad$ PIB por habitante (PIB): similar en ambos países. Representa el Producto Interior Bruto por habitante para el año 2014 (último año con datos disponibles por regiones para Finlandia). Para la comparación se debe tener en cuenta lo expuesto para el caso de la variable Gastos del análisis por municipios. Los datos para Finlandia se han obtenido a partir de las tablas concerniente a las cuentas e informes nacionales en la fuente Statistics Finland's PX-Web databases (Statfin, 2018). Para España, de la sección de nombre Economía del Instituto $\mathrm{Na}$ cional de Estadística (INE, 2018).

Los resultados o descripción estadística de las variables incluidas en el estudio por regiones se muestran en la Tabla II.

Como se puede observar, nuevamente las medias son mayores en Finlandia que en España y tan solo menores en las variables donde el mejor resultado sería el más bajo (Habitantes, Educación y Desempleo). A su vez, los resultados del porcentaje de la variación de la desviación típica siempre son menores en Finlandia que en España, confirmando la idea ya expuesta de que las bibliotecas públicas son más homogéneas en el primer país y sin tantas diferencias o desigualdades como en España tanto a nivel de municipios como por regiones o comunidades autónomas. Desde ahí, adquiere aún un mayor sentido la idea sobre la posibilidad y necesidad de una ley a nivel nacional para la mejora y la superación de dichas diferencias en España.

Cabe subrayar que en el análisis por regiones la variable Actividades resulta con una media mayor para Finlandia en comparación a España, algo que no sucedía en el análisis por municipios. Esto se debe, principalmente, a que el total de la población en el análisis por regiones para España varía y aumenta un $7,60 \%$ respecto del análisis por municipios (de 42.860 .838 a 46.386 .463 ), y en Finlandia tan solo un $0,07 \%$ (de 5.438 .855 a 5.442.837). La población total considerada en el estudio por regiones para ambos casos es la total de cada región y de los países, mientras que para el estudio por municipios resulta de la suma de la población de los municipios con servicios de bibliotecas y con datos disponibles en la fuente empleada. A su vez, las diferencias de los resultados de las variables en los análisis por municipios y regiones se deben a que en el segundo caso no se realiza la limpieza de datos de municipios con resultado de 0 en las variables que sí se llevó a cabo en el análisis a dicho nivel, siendo los resultados para cada región o comunidad autónoma la suma de todos los municipios con datos disponibles en cada una de ellas y la población total la de cada región.

Como ya se expuso pero conviene recordar, la ausencia de datos supuso eliminar 368 municipios de España del total de 3312 con bibliotecas, según la fuente, resultando un tamaño para la muestra de 2944 ( $n=2944)$. Para el caso de Finlandia, solo se eliminó un municipio por ausencia de datos para la Superficie y por ello la diferencia de población entre el análisis por regiones y por municipios es mínima (0,07\% menos en el segundo caso), resultando un tamaño de la muestra de $282(n=282)$. El tamaño de la muestra por regiones de Finlandia es de $18(n=18)$ y en España de $17(n=17)$.

Tabla II. Descripción estadística de las variables a nivel de regiones

\begin{tabular}{|l|c|c|c|c|c|c|}
\hline \multirow{2}{*}{ Variable } & \multicolumn{3}{|c|}{ Finlandia $\mathbf{n} \mathbf{1 8}$} & \multicolumn{3}{c|}{ España $\mathbf{~} \mathbf{1 7}$} \\
\cline { 2 - 7 } & Media & sd & \% variación sd & Media & sd & \% variación sd \\
\hline Gastos & 38,7 & 2,9 & $7,4 \%$ & 7,4 & 2,4 & $32,2 \%$ \\
\hline Colección & 8,1 & 1,7 & $21,5 \%$ & 2,1 & 0,8 & $35,4 \%$ \\
\hline Adquisiciones & 36,4 & 4,8 & $13,1 \%$ & 7,8 & 2,1 & $26,6 \%$ \\
\hline Actividades & 7,3 & 1,9 & $26,1 \%$ & 4,3 & 3,6 & $84,6 \%$ \\
\hline Superficie & 103,1 & 14,2 & $13,8 \%$ & 36,8 & 13,5 & $36,8 \%$ \\
\hline Personal & 3,8 & 0,3 & $7,9 \%$ & 1,2 & 0,3 & $24,6 \%$ \\
\hline Profesionales & 0,4 & 0,1 & $18,3 \%$ & 0,2 & 0,1 & $31,6 \%$ \\
\hline Habitantes & 6851,8 & 2458,7 & $35,9 \%$ & 10901,7 & 6444,9 & $59,1 \%$ \\
\hline Préstamos & 16,8 & 1,5 & $9,1 \%$ & 1,1 & 0,5 & $42,8 \%$ \\
\hline Visitas & 8,7 & 0,8 & $9,3 \%$ & 2,5 & 0,7 & $27,9 \%$ \\
\hline PIB & 33381,4 & 4830,8 & $14,5 \%$ & 21950,6 & 4661,4 & $21,2 \%$ \\
\hline Educación & 15,8 & 1,8 & $11,3 \%$ & 42,3 & 7,8 & $18,5 \%$ \\
\hline Desempleo & 9,9 & 2,1 & $20,8 \%$ & 22,5 & 5,6 & $24,8 \%$ \\
\hline
\end{tabular}

Nota: sd equivale a desviación típica; \% variación sd se obtiene de la división de sd entre la media, multiplicado por 100. 


\section{RESULTADOS}

\subsection{Modelizando los Préstamos en Finlandia (nivel municipios)}

La estimación paso a paso produjo un modelo con 5 variables significativas contribuyendo a unos mayores Préstamos. El modelo explicó el 23\% de la variación en los Préstamos (Tabla III). Las variables Actividades $(\beta=0,48 ; p=0,433)$, Personal ( $\beta$ $=0,079 ; p=0,490)$, Profesionales $(\beta=-0,090 ; p$ $=0,109)$, fueron excluidas del modelo debido a la falta de significancia. Resulta interesante que ninguna de las variables relacionadas directamente con el personal resulten ser factores significativos para los Préstamos.

Los 5 predictores que se hallaron fueron los referidos a las variables Habitantes $(\beta=0,296)$, Adquisiciones $(\beta=0,341)$, Colección $(\beta=-0,286)$, Gastos $(\beta=0,282)$, y Superficie $(\beta=-0,214)$. Aunque en el Paso 5 el coeficiente beta es mayor para las Adquisiciones que para los Habitantes, este último predictor es el más fuerte de acuerdo a los resultados del coeficiente de determinación (R2), pues explica una mayor variación en los Préstamos ( $9 \%$ del Paso 1). El predictor para las Adquisiciones añade un $5,5 \%$ al anterior porcentaje en el Paso 2. Juntos, estos dos predictores explican una variación del 14,5\% en los Préstamos (Paso 2). Con todo, el modelo indica que a mayor Habitantes, Adquisiciones y Gastos, y menor Colección y Superficie, mayores son los Préstamos.

Cabe resaltar que la Superficie como predictor con valor negativo se ha dado en estudios anteriores (Japzon y Gong, 2005), pero sin un análisis concreto de los posibles motivos. Desde ahí, se propone una explicación para los casos con coeficiente beta negativo de la Colección y la Superficie en el modelo. Para ello, se atiende a la correlación parcial entre la Colección y Préstamos, y a la de Superficie y Préstamos, considerando a la población total (Población) como variable de control (Tabla IV).

Como se puede observar, la correlación sin variable de control entre Población y los Préstamos es positiva y significativa $\left(0,164^{* *}\right)$, y las correlaciones igualmente sin variable de control tanto entre Población y Colección $(-0,362 * * *)$, y Población y Superficie $(-0,284 * * *)$, son negativas y también significativas. A su vez, las correlaciones con la va-

Tabla III. Regresión lineal múltiple para los préstamos en Finlandia

\begin{tabular}{|l|l|c|c|c|c|c|}
\hline \multicolumn{1}{|c|}{ Paso } & \multicolumn{1}{|c|}{ Variable Introducida } & $\boldsymbol{\beta}$ & $\boldsymbol{\beta}$ & $\boldsymbol{\beta}$ & $\boldsymbol{\beta}$ & $\boldsymbol{\beta}$ \\
\hline 1. & Habitantes & $0,300^{* * *}$ & $0,432^{* * *}$ & $0,349^{* * *}$ & $0,334^{* * *}$ & $0,296^{* * *}$ \\
\hline 2. & Adquisiciones & & $0,269^{\star * *}$ & $0,420^{* * *}$ & $0,334^{\star * *}$ & $0,341^{* * *}$ \\
\hline 3. & Colección & & & $-0,278^{* * *}$ & $-0,380^{* * *}$ & $-0,286^{* *}$ \\
\hline 4. & Gastos & & & & $0,237^{* *}$ & $0,282^{* * *}$ \\
\hline 5. & Superficie & & & & & $-0,214^{* *}$ \\
\hline R & & 0,300 & 0,380 & 0,424 & 0,457 & 0,479 \\
\hline R2 & & 0,090 & 0,145 & 0,179 & 0,209 & 0,230 \\
\hline Adj. R2 & & 0,086 & 0,138 & 0,170 & 0,196 & 0,215 \\
\hline R2 change & & $0,090^{* * *}$ & $0,055^{* * *}$ & $0,035^{* * *}$ & $0,029^{* * *}$ & $0,021^{* * *}$ \\
\hline Model F & & $26,087^{* * *}$ & $16,799^{* * *}$ & $11,131^{* * *}$ & $9,590^{* *}$ & $7,129^{* *}$ \\
\hline
\end{tabular}

Nota: $n=282 .{ }^{*} p \leq 0,05 ; * * p \leq 0,01 ; * * * p \leq 0,001$.

Tabla IV. Correlaciones parciales para Finlandia

\begin{tabular}{|l|l|c|c|c|}
\hline \multicolumn{1}{|c|}{ Variables de control } & \multicolumn{1}{|c|}{ Variables } & Colección & Superficie & Préstamos \\
\hline \multirow{3}{*}{ Sin control } & Población & $-0,362 * * *$ & $-0,284 * * *$ & $0,164 * *$ \\
\cline { 2 - 5 } & Superficie & - & - & $-0,178 * *$ \\
\cline { 2 - 5 } & Colección & - & - & $-0,169 * *$ \\
\hline \multirow{2}{*}{ Población } & Colección & - & - & $-0,119 *$ \\
\cline { 2 - 5 } & Superficie & - & - & $-0,139 *$ \\
\hline
\end{tabular}

Nota: $n=282 .{ }^{*} p \leq 0,05 ; * * p \leq 0,01 ; * * * p \leq 0,001$. 
riable de la Población como control entre Colección y Préstamos $(-0,119 *)$ y entre Superficie y Préstamos $(-0,139 *)$ en ambos casos son negativas y significativas, pero los valores y la significancia son menores que en las correlaciones no controladas por la variable de la Población.

De todo ello se puede concluir que en los municipios con menor Colección y Superficie se están produciendo más Préstamos, lo que contradice en un cierto grado la hipótesis general acerca de la relación positiva entre un mayor abastecimiento (colección, superficie, etc) y uso de las bibliotecas. No obstante, puede ser que en los municipios con mayor Población, el tamaño de la superficie y la colección por habitante sea más pequeño, pero los préstamos por habitante sean mayores que en los municipios pequeños. Si lo anterior fuese cierto, controlando el tamaño de la Población en la relación entre las variables de Superficie y Colección con la de Préstamos, la relación negativa debería decrecer e incluso desaparecer. $Y$ eso es justo lo que sucede atendiendo a los resultados de las correlaciones parciales en Finlandia.

\subsection{Modelizando los Préstamos en España (nivel municipios)}

El mejor modelo consistió en 7 variables significativas contribuyendo a unos mayores Préstamos. Tan solo la variable Personal $(\beta=-0,023 ; p=0,303)$ fue excluida del modelo debido a la ausencia de significancia. En este caso, a diferencia del de Finlandia, las variables de Actividades y Profesionales resul- taron predictores. El modelo explicó el 16,8\% de la variación en los Préstamos (Tabla V), algo menos que en el caso de Finlandia (23\%).

La Colección resultó el predictor más fuerte ( $\beta=$ $0,393)$ para los Préstamos en comparación a la Superficie $(\beta=-0,231)$, las Actividades $(\beta=0,142)$, los Habitantes $(\beta=0,126)$, los Gastos $(\beta=0,089)$, las Adquisiciones $(\beta=0,060)$ y la variable Profesionales $(\beta=0,057)$. Al respecto, destacan las 3 primeras variables mencionadas por su buena contribución a los Préstamos, explicando el 14\% de su variación (Paso 3). La contribución de las restantes variables es menor, aportando un 2,8\% más a dicha variación en los Préstamos (Paso 7).

Este modelo indica que a mayor Colección, Actividades, Habitantes, Gastos, Adquisiciones y Profesionales, y menor Superficie, mayores son los Préstamos. Cabe resaltar que para España se obtuvieron 2 nuevos factores como predictores respecto del modelo para los Préstamos en Finlandia, a saber, los referidos a las variables Actividades y Profesionales. Además, el predictor más fuerte para los Préstamos en el caso español resultó el de la Colección, mientras que para Finlandia fue el de Habitantes.

Cabe destacar también que el coeficiente beta para la Colección en España, a diferencia del caso de Finlandia, resultó con signo positivo. A su vez, la Superficie sí que resultó predictor negativo, tal y como sucedió con Finlandia. Al respecto, se ha calculado la correlación parcial entre Superficie y Préstamos con la Población como variables de control (Tabla VI).

Tabla V. Regresión lineal múltiple para los Préstamos en España

\begin{tabular}{|l|l|c|c|c|c|c|c|c|}
\hline Paso & $\begin{array}{c}\text { Variable } \\
\text { Introducida }\end{array}$ & $\boldsymbol{\beta}$ & $\boldsymbol{\beta}$ & $\boldsymbol{\beta}$ & $\boldsymbol{\beta}$ & $\boldsymbol{\beta}$ & $\boldsymbol{\beta}$ & $\boldsymbol{\beta}$ \\
\hline 1. & Colección & $0,301 * * *$ & $0,421 * * *$ & $0,384 * *$ & $0,437 * *$ & $0,404 * * *$ & $0,386 * *$ & $0,393 * * *$ \\
\hline 2. & Superficie & & $-0,210 * * *$ & $-0,214 * * *$ & $-0,233 * * *$ & $-0,237 * * *$ & $-0,230 * * *$ & $-0,231^{* * *}$ \\
\hline 3. & Actividades & & & $0,145 * * *$ & $0,154 * * *$ & $0,144 * * *$ & $0,142 * * *$ & $0,142 * * *$ \\
\hline 4. & Habitantes & & & & $0,131 * * *$ & $0,128 * * *$ & $0,130 * * *$ & $0,126 * * *$ \\
\hline 5. & Gastos & & & & & $0,094 * * *$ & $0,092 * * *$ & $0,089 * * *$ \\
\hline 6. & Adquisiciones & & & & & & $0,062 * * *$ & $0,060 * * *$ \\
\hline 7. & Profesionales & & & & & & & $0,057 * * *$ \\
\hline R & & 0,301 & 0,347 & 0,374 & 0,393 & 0,402 & 0,406 & 0,410 \\
\hline R2 & & 0,090 & 0,120 & 0,140 & 0,154 & 0,162 & 0,165 & 0,168 \\
\hline Adj. R2 & & 0,090 & 0,119 & 0,139 & 0,153 & 0,160 & 0,163 & 0,166 \\
\hline $\begin{array}{l}\text { R2 } \\
\text { change }\end{array}$ & & $0,090 * * *$ & $0,030 * * *$ & $0,020 * * *$ & $0,015 * * *$ & $0,007 * * *$ & $0,004 * * *$ & $0,003 * * *$ \\
\hline Model F & & $292,165 * * *$ & $99,100 * * *$ & $66,642 * * *$ & $51,185 * * *$ & $25,778 * * *$ & $12,363 * * *$ & $11,283 * * *$ \\
\hline
\end{tabular}

Nota: $n=2944 . * p \leq 0,05 ; * * p \leq 0,01 ; * * * p \leq 0,001$. 
Tabla VI. Correlación parcial entre Superficie y Préstamos España

\begin{tabular}{|l|l|c|c|}
\hline \multicolumn{1}{|c|}{ Variables de control } & Variable & Superficie & Préstamos \\
\hline \multirow{2}{*}{ Sin control } & Población & $-0,028$ & 0,024 \\
\cline { 2 - 4 } & Superficie & - & 0,031 \\
\hline Población & Superficie & - & 0,031 \\
\hline
\end{tabular}

Nota: $n=2944 .{ }^{*} p \leq 0,05 ; * * p \leq 0,01 ; * * * p \leq 0,001$.

Tal y como se puede ver, no se halló significancia en las correlaciones. Así pues, en el caso de España el tamaño de la Población no influye en los resultados de la correlación entre Superficie y Préstamos, algo que sí ocurría en el caso de Finlandia.

\subsection{Modelizando las Visitas en Finlandia (nivel municipios)}

La estimación paso a paso produjo un modelo con 3 variables significativas contribuyendo a unas mayores Visitas, explicando el $18 \%$ de su variación (Tabla VII), resultado menor que para el modelo de los Préstamos en Finlandia (23\%).

Las variables Colección ( $\beta=-0,012 ; p=0,894)$, Superficie $(\beta=-0,005 ; p=0,952)$, Personal $(\beta=$ $0,124 ; p=0,329)$, Profesionales $(\beta=0,056 ; p=$ $0,322)$ y Adquisiciones ( $\beta=-0,012 ; p=0,880)$, fueron excluidas del modelo debido a la ausencia de significancia. Los Gastos resultaron el predictor más fuerte para las Visitas, pues su contribución ( $\beta$ $=0,341)$ es mayor que los casos de Actividades $(\beta$ $=0,217)$ y Habitantes $(\beta=0,181)$.

En este caso y a diferencia del modelo para los Préstamos en Finlandia, ninguno de estos predictores resulta con un valor negativo en el coeficiente beta. Por tanto, el modelo indica que a mayores Gastos, Actividades y Habitantes, mayores son las
Visitas. A su vez, atendiendo a los predictores resultantes para los Préstamos y Visitas en Finlandia, solo los Gastos resultaron como predictor en ambos casos, mientras que los referidos a Actividades y Habitantes resultaron predictores para las Visitas sin serlo para los Préstamos.

\subsection{Modelizando las Visitas en España (nivel municipios)}

El mejor modelo consistió en las 8 variables independientes consideradas al resultar todas ellas significativas y contribuyendo a unas mayores Visitas. El modelo explicó el $17 \%$ de la variación en las Visitas (Tabla VIII), resultado cercano al caso del modelo para las Visitas en Finlandia (18\%) y al del modelo de los Préstamos en España (16,8\%).

El predictor más fuerte resultó ser la Colección $(\beta=0,314)$ frente a las Actividades $(\beta=0,177)$, Habitantes $(\beta=0,147)$, Superficie $(\beta=-0,167)$, Personal $(\beta=0,094)$, Gastos $(\beta=0,079)$, Profesionales $(\beta=0,052)$ y Adquisiciones $(\beta=0,043)$. Los 5 primeros predictores mencionados destacan por su buena contribución a las Visitas, explicando el $16,8 \%$ de su variación (Paso 5). La contribución de las restantes variables es menor, aportando un $0,9 \%$ más a dicha variación (Paso 8).

Tabla VII. Regresión lineal múltiple para las Visitas en Finlandia

\begin{tabular}{|l|l|c|c|c|}
\hline \multicolumn{1}{|c|}{ Paso } & \multicolumn{1}{|c|}{ Variable Introducida } & $\boldsymbol{\beta}$ & $\boldsymbol{\beta}$ & $\boldsymbol{\beta}$ \\
\hline 1. & Gastos & $0,344 * * *$ & $0,284 * * *$ & $0,341^{* * *}$ \\
\hline 2. & Actividades & & $0,193 * * *$ & $0,217 * * *$ \\
\hline 3. & Habitantes & & & $0,181^{* *}$ \\
\hline R & & 0,344 & 0,390 & 0,424 \\
\hline R2 & & 0,118 & 0,152 & 0,180 \\
\hline Adj. R2 & & 0,115 & 0,145 & 0,170 \\
\hline R2 change & & $0,118 * * *$ & $0,034 * * *$ & $0,028 * * *$ \\
\hline Model F & & $35,536 * * *$ & $10,432 * * *$ & $8,934 * *$ \\
\hline
\end{tabular}

Nota: $n=282 . * p \leq 0,05 ; * * p \leq 0,01 ; * * * p \leq 0,001$. 
Tabla VIII. Regresión lineal múltiple para las Visitas en España

\begin{tabular}{|l|l|c|c|c|c|c|c|c|c|}
\hline Paso & $\begin{array}{c}\text { Variable } \\
\text { Introducida }\end{array}$ & $\boldsymbol{\beta}$ & $\boldsymbol{\beta}$ & $\boldsymbol{\beta}$ & $\boldsymbol{\beta}$ & $\boldsymbol{\beta}$ & $\boldsymbol{\beta}$ & $\boldsymbol{\beta}$ & $\boldsymbol{\beta}$ \\
\hline 1. & Colección & $0,305 * * *$ & $0,253^{* * *}$ & $0,292^{* * *}$ & $0,388^{* * *}$ & $0,336 * * *$ & $0,319 * * *$ & $0,324 * * *$ & $0,314 * * *$ \\
\hline 2. & Actividades & & $0,195 * * *$ & $0,203 * * *$ & $0,207 * * *$ & $0,181 * * *$ & $0,178 * * *$ & $0,177 * * *$ & $0,177 * * *$ \\
\hline 3. & Habitantes & & & $0,123 * * *$ & $0,141 * * *$ & $0,154 * * *$ & $0,149 * * *$ & $0,146 * * *$ & $0,147 * * *$ \\
\hline 4. & Superficie & & & & $-0,159 * * *$ & $-0,171 * * *$ & $-0,172 * * *$ & $-0,173 * * *$ & $-0,167 * * *$ \\
\hline 5. & Personal & & & & & $0,123 * * *$ & $0,097 * * *$ & $0,100 * * *$ & $0,094 * * *$ \\
\hline 6. & Gastos & & & & & & $0,082 * * *$ & $0,079 * * *$ & $0,079 * * *$ \\
\hline 7. & Profesionales & & & & & & & $0,054 * *$ & $0,052 * *$ \\
\hline 8. & Adquisiciones & & & & & & & & $0,043 *$ \\
\hline R & & 0,305 & 0,358 & 0,376 & 0,398 & 0,409 & 0,416 & 0,419 & 0,421 \\
\hline R2 & & 0,093 & 0,128 & 0,141 & 0,158 & 0,168 & 0,173 & 0,176 & 0,177 \\
\hline Adj. R2 & & 0,093 & 0,128 & 0,141 & 0,157 & 0,166 & 0,171 & 0,174 & 0,175 \\
\hline $\begin{array}{l}\text { R2 } \\
\text { change }\end{array}$ & & $0,093 * * *$ & $0,035 * * *$ & $0,013 * * *$ & $0,017 * * *$ & $0,009 * * *$ & $0,005 * * *$ & $0,003 * * *$ & $0,002 * * *$ \\
\hline Model F & & $300,938 * * *$ & $118,958 * * *$ & $45,561 * * *$ & $58,713 * * *$ & $32,854 * * *$ & $18,492 * * *$ & $10,005 * *$ & $5,900 *$ \\
\hline
\end{tabular}

Nota: $n=2944 . * p \leq 0,05 ; * * p \leq 0,01 ; * * * p \leq 0,001$.

Con todo, el modelo indica que a mayor Colección, Actividades, Habitantes, Personal, Gastos, Profesionales y Adquisiciones, y menor Superficie, mayores son las Visitas. A diferencia del modelo para las Visitas en Finlandia en el que se obtuvieron los predictores de Gastos, Actividades y Habitantes, para el caso de España resultaron como predictores todos los considerados como variables independientes, siendo el más fuerte el de la Colección, tal y como sucedió para el modelo de los Préstamos también en España. La Colección no resultó predictor para el caso en Finlandia de las Visitas, donde el más fuerte fue el de los Gastos.

Resulta de nuevo interesante el resultado negativo de la Superficie como predictor en el modelo de las Visitas en España, algo que ya ocurrió en los modelos para los Préstamos tanto en Finlandia como en España. Tal y como se hizo en esos casos, se ha estudiado la correlación parcial considerando a la Población como variable de control (Tabla IX).

Como se puede comprobar, no se obtuvo significancia entre la Población y la Superficie $(-0,028)$ y entre la Población y las Visitas $(0,016)$. Por ello, los resultados para la correlación entre Superfi- cie y Visitas tanto no controlada como controlada por la Población resultan similares (de 0,084*** y $0,085^{* * *}$ respectivamente). Tal y como sucedió con la correlación parcial en el modelo de los Préstamos en España, en este caso tampoco la Población influye en los resultados para la correlación entre Superficie y Visitas, algo que sí ocurrió en el caso de los Préstamos en Finlandia.

\subsection{Modelizando los Préstamos y las Visitas por Regiones}

En el estudio por regiones se analizaron los modelos para las variables dependientes de Préstamos y Visitas en ambos países, encontrando tan solo un predictor para los dos modelos en España de los Préstamos y las Visitas. Dado que para Finlandia no se hallaron predictores en ninguno de los modelos para los Préstamos y las Visitas, y que el predictor para ambos modelos en España es el mismo, se ha simplificado la presentación de los resultados (Tabla $\mathrm{X}$ ).

La estimación paso a paso para los Préstamos en España produjo un modelo con la variable Ad-

Tabla IX. Correlación parcial Superficie-Visitas España

\begin{tabular}{|l|l|c|c|}
\hline \multicolumn{1}{|c|}{ Variables de control } & \multicolumn{1}{c|}{ Variables } & Superficie & Visitas \\
\hline Sin control & Población & $-0,028$ & 0,016 \\
\hline & Superficie & - & $0,084 * * *$ \\
\hline Población & Superficie & - & $0,085 * * *$ \\
\hline
\end{tabular}

Nota: $n=2944 .{ }^{*} p \leq 0,05 ; * * p \leq 0,01 ; * * * p \leq 0,001$. 
Tabla X. Regresiones múltiples para España por regiones

\begin{tabular}{|l|c|c|c|}
\hline \multicolumn{1}{|c|}{ Paso } & \multicolumn{1}{c|}{ Variable Introducida } & $\boldsymbol{\beta}$ Modelo Préstamos & $\boldsymbol{\beta}$ Modelo Visitas \\
\hline 1. & Adquisiciones & $0,852 * * *$ & $0,749 * * *$ \\
\hline R & & 0,85 & 0,75 \\
\hline R2 & & 0,73 & 0,53 \\
\hline Adj. R2 & & 0,71 & $0,562 * * *$ \\
\hline R2 change & & $39,882 * * *$ & $19,215 * * *$ \\
\hline Model F & & & \\
\hline
\end{tabular}

Nota: $n=17 . * p \leq 0,05 ; * * p \leq 0,01 ; * * * p \leq 0,001$.

quisiciones ( $\beta=0,852$ ), explicando el $73 \%$ de la variación en los Préstamos. Por otro lado, la estimación paso a paso para las Visitas en el mismo país produjo un modelo con la variable Adquisiciones ( $\beta$ $=0,749$ ), explicando el $56 \%$ de la variación en las Visitas. Por tanto, las variables del entorno consideradas en el análisis por regiones (PIB, Educación y Desempleo) y todas las consideradas en el análisis por municipios a excepción de las Adquisiciones en el caso de España, fueron excluidas de los modelos.

\section{DISCUSIÓN}

El presente estudio ofrece información sobre los sistemas de bibliotecas públicas de España y Finlandia. Los resultados están basados en datos para el año 2015. A partir del análisis estadístico de las medias y las desviaciones típicas tanto de características propias de las bibliotecas como de algunas referidas al entorno socioeconómico, se ha comprobado que en España se da una mayor desigualdad frente a la mayor homogeneidad de las de Finlandia, tanto a nivel de municipios como por regiones. Además, el estudio ofrece información sobre los factores para un mayor uso de las bibliotecas entendido desde los préstamos y las visitas. Se ha trabajado por modelizar el uso de las bibliotecas públicas en base a análisis de regresiones lineales múltiples con la técnica paso a paso. Con todo, este es el primer estudio en que se lleva a cabo la modelización del uso de las bibliotecas públicas españolas. Además, su originalidad radica también en que se comparan los resultados con respecto a Finlandia, país modelo de buenas prácticas en lo relacionado a bibliotecas públicas.

En cuanto a las diferencias en torno a la homogeneidad de las bibliotecas públicas de España y Finlandia, cabe subrayar lo que se especificó al respecto de la Library Act de 1961 de Finlandia. En esta ley se marcó el objetivo de superar las desigualdades entre las zonas rurales y urbanas del país (Mäkinen, 2001). El paso del tiempo demostró la eficacia en la consecución de los objetivos, en parte también gracias a los avances en lo referido al estado de bienestar, la educación y la cultura en general, acontecidos en el mismo periodo. Se pasó de unos préstamos por habitante de 1,5 en 1950 a los 19,9 de 1990, siendo el crecimiento mayor en las zonas rurales que en las urbanas. La falta de homogeneidad en España puede entenderse desde la ausencia e ineficacia de leyes de ámbito nacional que regulen la realidad de las bibliotecas para su transformación hacia un mayor uso y mayores beneficios para la sociedad. (BOE, 2017).

Por otro lado, los factores que resultaron predictores en los modelos para los Préstamos y las Visitas a nivel de municipios varían de un país a otro. En España, de las 8 variables consideradas como independientes, fueron 7 y 8 las que resultaron predictores para los Préstamos y las Visitas respectivamente, siendo tan solo el referido al Personal el que no resultó como predictor en el modelo de los Préstamos. En cuanto a Finlandia, se hallaron 5 predictores para los Préstamos (Habitantes, Adquisiciones, Colección, Gastos, Superficie) y 3 para las Visitas (Gastos, Actividades, Habitantes). A su vez, el predictor referido a Habitantes resultó como más fuerte para los Préstamos en Finlandia, y el de la Colección para España. Para las Visitas, el predictor más fuerte en Finlandia fue el de Gastos, mientras que en España resultó nuevamente el de Colección. Por otro lado, a nivel de regiones, tan solo se halló el factor de Adquisiciones como predictor para España en los modelos para los Préstamos y las Visitas, sin resultar predictores ninguno de los factores considerados como añadidos en dicho nivel respecto del análisis por municipios (PIB, Educación y Desempleo).

En un ejercicio de comparación de los resultados de este estudio con los de otros vistos en la revisión bibliográfica, cabe destacar que las variables del entorno socioeconómico que se han considerado en el análisis por regiones (Educación, PIB, Desempleo), no resultaron tampoco predictores con significancia en algunos de ellos (Yilmaz, 1998; Lara y otros, 
2015). Pese a ello, son muchos los estudios en que dichas variables sí que resultaron significantes en relación al uso de las bibliotecas (Creaser y Sumsion, 1995; Yilmaz, 1998; Smith, 1999; Japzon y Gong, 2005; Uddin y otros, 2006; Burke, 2007; SeiChing y Kyung-Sun, 2008; Lara y otros, 2015). A su vez, en nuestro estudio las variables referidas al PIB y Educación no resultaron predictores, mientras que en algunos de los revisados sí que se encontró relación significativa y negativa con los préstamos (Huysmans y Hillebrink, 2008). Al respecto, los autores de este último estudio afirman que los préstamos pueden verse reducidos por el aumento de la prosperidad y la posibilidad de gastar en la compra de libros en detrimento de tomar materiales prestados de las bibliotecas.

Los casos de los predictores Superficie y Colección con valor negativo en el coeficiente beta se analizaron y trataron de explicarse mediante correlaciones parciales considerando a la Población total como variable de control. Concretamente, los predictores Superficie y Colección resultaron con valor negativo en el modelo para los Préstamos de Finlandia a nivel de municipios, y el de Superficie en los modelos para Préstamos y visitas en España, también a nivel de municipios. En ese sentido, cabe destacar que en la literatura revisada se encontró un caso en el que se obtenía también un valor negativo en el coeficiente beta para la superficie (Japzon y Gong, 2005).

Al respecto, para explicar los valores negativos en este trabajo, se propuso la influencia del tamaño de la población total como variable de control. Concretamente, en el caso de Finlandia se verificó la influencia del tamaño de la Población en los casos de la relación negativa entre Superficie y Préstamos, y entre la relación también negativa entre Colección y Préstamos. Por otro lado, para los casos de España se comprobó que la población total no ejercía una influencia significativa como variable de control entre las relaciones de Superficie y Préstamos, y la de Superficie y Visitas. Aún con todo, resulta interesante remarcar que las variables Superficie y Colección guardan una relación negativa con los Préstamos y las Visitas en los modelos desarrollados en el presente estudio y en los casos mencionados.

Por último, los modelos a nivel de municipios explicaron el $23 \%$ de los Préstamos y el $18 \%$ de las Visitas en Finlandia, y el $16,8 \%$ y $17 \%$ respectivamente para España, por lo que, aproximadamente, más del $75 \%$ de la variación de sendas variables se explica mediante otros factores. Desde ahí, es importante marcar la necesidad de futuras investigaciones para la búsqueda de los factores que permitan predecir los modelos. Una opción sería la de ampliar este tipo de estudios a las variables que se incluyen en los tra- bajos en la literatura revisada, algo que para España será igualmente original, valioso, y posible, siempre y cuando se disponga de datos para ello.

\section{CONCLUSIONES}

Este estudio puede ser considerado como uno de los primeros sobre la modelización del uso de las bibliotecas públicas españolas a través de sus características y también como uno de los primeros en poner en relación los resultados con los de otro país, en este caso Finlandia. En base al estudio se puede concluir que las bibliotecas de Finlandia se caracterizan por una mayor homogeneidad tanto a nivel de municipios como por regiones frente a la existencia de grandes diferencias en las de España. El hecho de comparar países ha permitido obtener información valiosa y original para el caso español. Además, el estudio ofrece también información sobre los factores que predicen el uso de las bibliotecas públicas considerado como los préstamos y las visitas a las mismas. Con todo, se han satisfecho las dos preguntas de investigación que se marcaron al inicio del estudio.

Tal y como se vio en los resultados y se analizó en la discusión, los modelos alcanzados en el análisis a nivel de municipios explican desde el $16 \%$ hasta el $23 \%$ de la variación en el uso de las bibliotecas públicas. Los predictores para cada país y modelo variaron y se analizaron en la discusión. Lo que sí conviene resaltar aquí es que para España el predictor más fuerte, tanto para Préstamos como para Visitas, resultó ser el referido a la variable Colección, mientras que para Finlandia fueron los referidos a los Habitantes y a los Gastos, respectivamente. Desde ahí, cabe subrayar que en la discusión se presentaron los predictores para cada modelo y país, comparando lo obtenido en este estudio con la literatura revisada para su elaboración.

Cabe resaltar también como parte de conclusiones que este trabajo gana en originalidad al ofrecer una posible explicación de los predictores de valor negativo con el estudio de las correlaciones parciales y tomando a la Población como variable de control. No obstante, resulta interesante ya de por sí que tanto la Superficie como la Colección resulten como factores con relación negativa en su relación con los Préstamos y las Visitas, especialmente el caso de la Superficie, que se dio en los modelos para los Préstamos de Finlandia y en el de los Préstamos y las Visitas para España.

Por otro lado, en el análisis a nivel de regiones se incluyeron 3 variables del contexto socioeconómico no consideradas en el análisis por municipios (PIB, Educación y Desempleo). Estos nuevos factores no resultaron predictores para los modelos sobre el 
uso de las bibliotecas públicas en España y Finlandia. En ese sentido, una de las limitaciones del estudio se explica por la falta de disponibilidad de datos a nivel de municipios para variables relacionadas con el entorno socioeconómico y geográfico.

Con todo y de cara a futuros estudios, el hecho de que los modelos en el análisis a nivel de municipios no expliquen más del $75 \%$ de la variación (del $16 \%$ al $23 \%$ ), marca la línea para futuras investigaciones en aras a la identificación de otros factores que puedan aumentar la capacidad predictiva de los modelos. Una opción para ello sería precisamente la de considerar más variables del entorno socioeconómico y geográfico y otras no consideradas relacionadas con las características propias de las bibliotecas. Todo ello podría hacerse siempre en virtud de la disponibilidad de datos, para lo que se podría tratar de conseguir que los órganos gestores y administrativos los dispongan para poder ser utilizados en estudios como los

\section{REFERENCIAS}

Aabo, S.; Audunson, R. (2012). Use of library space and the library as place. Library \& Information Science Research, 34 (2), 138-149. https://doi.org/10.1016/j. lisr.2011.06.002

Aabo, S.; Audunson, R.; Varheim, A. (2010). How do public libraries function as meeting places? Library \& Information Science Research, 32 (1), 16-26. https:// doi.org/10.1016/j.lisr.2009.07.008

BOE. (2017). Código de Legislación Bibliotecaria. Selección y ordenación: ANABAD. Códigos Electrónicos. Boletín Oficial del Estado. [En línea]. Disponible en: http://www.boe.es/legislacion/codigos/abrir_pdf. php?fich=024_Codigo_de_Legislacion_Bibliotecaria. pdf [Fecha de consulta: 18/09/2018].

Burke, S. K. (2007). The use of public libraries by native Americans. Library Quarterly, 77 (4), 429-461. https://doi.org/10.1086/520998

Carrión-Gútiez, A. (2013). Informe de situación de las bibliotecas públicas españolas en 2012. El Profesional de la Información, 22 (3), 250-258. https://doi. org/10.3145/epi.2013.may.09

Chen, P.; Rea, C.; Shaw, R.; Bottino, C. J. (2016). Associations between Public Library Use and Reading Aloud among Families with Young Children. Journal of Pediatrics, 173, 221-227. https://doi.org/10.1016/j. jpeds.2016.03.016

CIS. (2014). Barómetro de diciembre 2014. Estudio no 3047. Centro de Investigaciones Sociológicas. [En línea]. Disponible en: http://www.cis.es/cis/export/ sites/default/-Archivos/Marginales/3040_3059/3047/ es3047mar.html [Fecha de consulta: 18/09/2018]. que se plantean. Con ello, se alcanzaría un mayor conocimiento de la realidad de las bibliotecas públicas españolas, lo que facilitaría su mejora y el aumento tanto del uso como de los beneficios a la sociedad.

\section{AGRADECIMIENTOS}

El primer autor es beneficiario de una de las ayudas para contratos predoctorales de Formación de Profesorado Universitario FPU-2014 del Ministerio de Educación, Cultura y Deporte de España ( $n^{0}$ de referencia FPU2014/04213).

\section{ACKNOWLEDGEMENTS}

The first signing author is a beneficiary of one of the grants for pre-doctoral contracts from the University Teacher Training Program FPU-2014 of the Ministry of Education, Culture and Sports of Spain (reference number FPU2014/04213).

CIS. (2016). Barómetro de junio 2016. Estudio no 3142. Centro de Investigaciones Sociológicas. [En línea]. Disponible en: http://www.cis.es/cis/export/sites/default/Archivos/Marginales/3140_3159/3142/Es3142mar. html [Fecha de consulta: 18/09/2018].

Creaser, C.; Sumsion, J. (1995). Affluence and public library use: The DoE Index of Local Conditions and library use in London and the metropolitan districts of England. Library Management, 16 (6), 3-15. https:// doi.org/10.1108/01435129510091793

D’Elia, G. ; Jörgensen, C. ; Woelfel, J.; Rodger, E. J. (2002). The impact of the Internet on public library use: An analysis of the current consumer market for library and Internet services. Journal of the American Society for Information Science and Technology, 53 (10), 802820. https://doi.org/10.1002/asi.10102

Delgado-López-Cozar, E. (2002). La investigación en biblioteconomía y documentación. Trea, Gijón, España.

Fernández-Ardèvol, M.; Ferran-Ferrer, N.; Nieto-Arroyo, J.; Fenoll, C. (2018). The public library as seen by the non-users. El profesional de la información, 27 (3), 659-670. https://doi.org/10.3145/epi.2018.may.19

Ferran-Ferrer, N.; Guallar, J.; Abadal, E.; Server, A. (2017). Research methods and techniques in Spanish library and information science journals (2012-2014). Information Research, 22 (1). http://www.informationr.net/ir/22-1/paper741.html

Gould, E.; Gomez, R. (2010). New challenges for libraries in the information age: a comparative study of ICT in public libraries in 25 countries. Information Development, 26 (2), 166-176. https://doi. org/10.1177/0266666910367739 
Guallar, J.; Ferran-Ferrer, N.; Abadal, E.; Server, A. (2017). Library and information science journals in Spain: Thematic and methodological analysis. El Profesional de la Información, 26 (5), 947-960. https:// doi.org/10.3145/epi.2017.sep.16

Hair, J. F.; Black, W. C.; Babin, B. J.; Anderson, R. E. (2010). Multivariate Data Analysis (7.a ed.). Upper Saddle River, New Jersey: Prentice-Hall Inc.

Herrera-Viedma, E.; López-Gijón, J. (2013). Libraries' social role in the information age. Science, 339 (6126), 1382. https://doi.org/10.1126/science.339.6126.1382-a

Houghton, K.; Foth, M.; Miller, E. (2013). The continuing relevance of the library as a third place for users and non-users of IT: the case of Canada Bay. The Australian Library Journal, 62 (1), 27-39. https://doi.org/10. 1080/00049670.2013.771764

Huysmans, F; Hillebrink, C. (2008). The future of the Dutch public library: ten years on. The Hague, The Netherlands: Netherlands Institute for Social Research.

Ignatow, G. (2011). What has globalization done to developing countries' public libraries? International Sociology, 26 (6), 746-768. https://doi. org/10.1177/0268580910393373

Ignatow, G.; Webb, S. M.; Poulin, M.; Parajuli, R.; Fleming, P.; Batra, S.; Neupane, D. (2012). Public Libraries and Democratization in Three Developing Countries: Exploring the Role of Social Capital. Libri, 62 (1), 67-80. https://doi.org/10.1515/libri-2012-0005

INE. (2018). Instituto Nacional de Estadística. [En línea]. Disponible en: http://www.ine.es/ [Fecha de consulta: 18/09/2018]

Japzon, A. C.; Gong, H. (2005). A neighborhood analysis of public library use in New York City. Library Quarterly, 75 (4), 446-463. https://doi.org/10.1086/502786

Johnson, C. A.; Griffis, M. R. (2014). The effect of public library use on the social capital of rural communities. Journal of Librarianship and Information Science, 46 (3), 179-190. https://doi. org/10.1177/0961000612470278

Jorgensen, C.; D'Elia, G.; Woelfel, J.; Rodger, E. (2001). The impact of the Internet on public library use: $\mathrm{Cu}$ rrent status and trends for the future. Proceedings of the ASIST Annual Meeting, 38, 141-155.

Keshvari, M.; Farashbandi, F. Z.; Geraei, E. (2015). Modelling influential factors on customer loyalty in public libraries: a study of West Iran. Electronic Library, 33 (4), 810-823. https://doi.org/10.1108/EL-10-2013-0185

Kim, G.; Yu, S. Y. (2011). An Exploratory Study to Develop an Alternative Model of Public Library Management Using the Institute of Museum and Library Services' Public Library Statistics. Library Quarterly, 81 (4), 359-382. https://doi.org/10.1086/661656

Kishida, K. (1998). A mathematical model on public library loan incorporating an inverse size effect. Library and Information Science, (40), 29-35.
Koontz, C. M.; Jue, D. K.; Lance, K. C. (2005). Neighborhood-based in-library use performance measures for public libraries: A nationwide study of majorityminority and majority white/low income markets using personal digital data collectors. Library and Information Science Research, 27 (1), 28-50. https://doi. org/10.1016/j.lisr.2004.09.003

Lai, H.-J.; Wang, C.-Y. (2012). Examining public librarian's information literacy, self-directed learning readiness, and e-learning attitudes: A study from Taiwan. Malaysian Journal of Library \& Information Science, 17 (2), 101-115.

Lara, L. S.; Stilwell, C.; Underwood, P. G. (2015). Correlations between the economy and public library use. Bottom Line, 28 (1-2), 26-33. https://doi.org/10.1108/ BL-12-2014-0032

Lázaro-Rodríguez, P.; López-Gijón, J.; Alonso, S.; Martínez-Sánchez, M.Á.; Herrera-Viedma, E. (2018a). Secaba-Rank, herramienta online para analizar y evaluar bibliotecas. El profesional de la información, 27 (2), 278-288. https://doi.org/10.3145/epi.2018.mar.06

Lázaro-Rodríguez, P; López-Gijón, J; Herrera-Viedma, E. (2018b). Visibilidad de las bibliotecas públicas y la lectura en medios de comunicación españoles frente a otros hechos de la cultura y relación con su uso: medidas para su mayor promoción y difusión. BiD: textos universitaris de biblioteconomia $i$ documentació, 40 (juny). http://dx.doi.org/10.1344/BiD2018.40.7

Lin, H.; Pang, N.; Luyt, B. (2015). Is the library a third place for young people? Journal of Librarianship and Information Science, 47 (2), 145-155. https://doi. org/10.1177/0961000614532303

Mäkinen, I. (2001). The golden age of Finnish public libraries: institutional, structural and ideological background since the 1960's. En: Mäkinen, Ilkka (editor) Finnish public libraries in the 20th century, pp. 116150. Tampere, Finland: Tampere University Press.

McClure, C. R.; Bertot, J. C. (1998). Public Library Use in Pennsylvania: Identifying Uses, Benefits, and Impacts. Final Report. Harrisburg, Pennsylvania: Pennsylvania State Dept. of Education.

MECD. (2015). Encuesta de Hábitos y Prácticas Culturales 2014-2015. Ministerio de Educación, Cultura y Deporte. [En línea]. Disponible en: http://es.calameo.com/ read/0000753358b572c390560 [Fecha de consulta: 18/09/2018].

MECD. (2018a). Bibliotecas públicas españolas en cifras. Ministerio de Educación, Cultura y Deporte. [En línea]. Disponible en: http://www.mecd.gob.es/culturamecd/areas-cultura/bibliotecas/mc/ebp/portada.html [Fecha de consulta: 18/09/2018].

MECD. (2018b). Base Estadísticas Educación. Ministerio de Educación, Cultura y Deporte. [En línea]. Disponible en: https://www.educacion.gob.es/educabase $/$ menu. .do?type= pcaxis\&path=/Formacionyml/ EPA2016/NivFor\&file $=$ pcaxis\& $\mathrm{I}=\mathrm{s0}$ [Fecha de consulta: 18/09/2018]. 
Obokoh, N. P.; Arokoyu, S. B. (1991). The influence of geographical location on public library use: a case study from a developing country. Bulletin - Special Libraries Association, Geography \& Map Division, 163, 30-42.

Sanderson, C. R. (1950). The Library's Public: A Report of the Public Library Inquiry. Bernard Berelson. The Library Quarterly, 20 (1), 47-49. https://doi. org/10.1086/617605

Sei-Ching, J. S; Kyung-Sun, K. (2008). Use and non-use of public libraries in the information age: A logistic regression analysis of household characteristics and library services variables. Library \& Information Science Research, 30 (3), 207-215. https://doi.org/10.1016/j. lisr.2007.11.008

Sei-Ching, J. S; Vakkari, P. (2015). Perceived outcomes of public libraries in the U.S. Library \& Information Science Research, 37 (3), 209-219. https://doi. org/10.1016/j.lisr.2015.04.009

Smith, I. M. (1999). What do we know about public library use? Aslib Proceedings, 51 (9), 302-314. https://doi. org/10.1108/EUM0000000006990

Statfin. (2018). Statistics Finland's PX-Web databases. [En línea]. Disponible en: http://pxnet2.stat.fi/PXWeb/ pxweb/en/StatFin/ [Fecha de consulta: 18/09/2018].

Sumsion, J.; Hawkins, M.; Morris, A. (2002). The economic value of book borrowing from public libraries - An optimisation model. Journal of Documentation, 58 (6), 662682. https://doi.org/10.1108/00220410210448200

SYKT. (2018). Suomen yleisten kirjastojen tilastot Finnish Public Libraries Statistics Database. [En línea]. Disponible en: http://tilastot.kirjastot.fi/index. php?lang=en [Fecha de consulta: 18/09/2018].

Uddin, M. N.; Quaddus, M.; Islam, M. S. (2006). Socio-economic-cultural aspects and mass information need: The case of public library uses in Bangladesh.
Library Management, 27 (9), 636-652. https://doi. org/10.1108/01435120610715536

Vakkari, P. (2012). Internet use increases the odds of using the public library. Journal of Documentation, 68 (5), 618638. https://doi.org/10.1108/00220411211256003

Vakkari, P. (2014). Models explaining the perceived outcomes of public libraries. Journal of Documentation, 70 (4), 640-657. https://doi.org/10.1108/JD-02-20130016

Vakkari, P.; Aabø, S. ; Audunson, R.; Huysmans, F.; Kwon, N.; Oomes, M.; Sin, S.-C. J. (2016). Patterns of perceived public library outcomes in five countries. Journal of Documentation, 72 (2), 342-361. https://doi. org/10.1108/JD-08-2015-0103

Vakkari, P.; Aabø, S.; Audunson, R.; Huysmans, F.; Oomes, M. (2014). Perceived outcomes of public libraries in Finland, Norway and the Netherlands. Journal of Documentation, 70 (5), 927-944. https://doi. org/10.1108/JD-06-2013-0072

Van-Ryzin, G. G.; Charbonneau, E. (2010). Public service use and perceived performance: an empirical note on the nature of the relationship. Public Administration, 88 (2), 551-563. https://doi.org/10.1111/j.14679299.2010.01820.x

Whitacre, B.; Rhinesmith, C. (2015). Public libraries and residential broadband adoption: Do more computers lead to higher rates? Government Information Quarterly, 32 (2), 164-171. https://doi.org/10.1016/j. giq. 2015.02 .007

Yilmaz, B. (1998). A sociological study of public library use in Ankara, Turkey. Journal of Librarianship and Information Science, 30 (4), 259-267. https://doi. org/10.1177/096100069803000406 